\title{
Strain Induced $k$-Linear Spin Splitting in III-V Semiconductors
}

\author{
A. Skierkowski AND J.A. MAJEWski \\ Institute of Theoretical Physics, Faculty of Physics, Warsaw University \\ Hoża 69, 00-681 Warszawa, Poland \\ We present theoretical studies of the linear- $k$ strain induced spin split- \\ ting of the conduction band in the zinc-blende semiconductors. The studies \\ are based on $a b$ initio calculations performed within the density functional \\ theory with non-scalar relativistic effects fully taken into account. This per- \\ mits one to construct effective Hamiltonian for the strain induced linear- $k$ \\ spin splitting of the zinc-blende semiconductors. This Hamiltonian repro- \\ duces fully the structure of the strain induced linear- $k$ spin splitting and \\ generalizes previously introduced and commonly used effective Hamiltonian.
}

PACS numbers: 71.70.Fk, 71.15.Rf, 71.70.Ej, 71.55.Eq

\section{Introduction}

An ability to control the spin transport in semiconductors is an essential issue in semiconductor spintronics and information processing $[1,2]$. One of the methods to achieve control of the electron spin degree of freedom involves the intrinsic spin-orbit interaction (SOI). The SOI can be tuned by means of electric field and/or electric gates [3] and this has been confirmed experimentally [4]. In semiconductors the spin-orbit effect appears as an interaction of the electron spin with an effective magnetic field, whose direction and magnitude depend on the electron momentum. The specific form of this dependence is determined by the crystal symmetry. Therefore, the form of SOI in semiconductors can be modified by deformation and can influence the spin current. Such effect of strain-induced SOI on spin transport has been demonstrated experimentally [5]. On the other hand, the strain can be associated with mechanical motion of solid, in particular, with oscillations in nanomechanical systems. This effect, in turn, facilitates the tuning of SOI with nanomechanical oscillations and allows the design of interesting devices [6]. Therefore, a detailed knowledge of the strain induced SOI in semiconductors is essential for understanding, modeling, and design of future functional devices. 
In the present paper, we focus on the strain-induced SOI in the conduction band. Traditionally, the strain effects on the spin splitting caused by SOI are described by the Hamiltonian introduced by Pikus and Titkov many years ago [7]. However, the microscopic analysis of the strain induced spin splitting is still lacking. In this paper, we study the spin splitting of the conduction band in zinc-blende semiconductors by means of ab initio calculations in the framework of the density functional theory (DFT) with non-scalar relativistic effects taken nonperturbatively into account. Our studies reveal new features of the SOI that are not included in the traditionally employed Hamiltonian. In particular, we find out that the SOI depends on the atomistic structural details of the strained semiconductor, and therefore cannot be described by the phenomenological Hamiltonian.

The paper is organized as follows. In Sect. 2, we describe very shortly the computational procedure, in Sect. 3 we discuss the obtained results, and finally we conclude the paper in Sect. 4.

\section{Calculational details}

The ab initio calculations of spin splitting in conduction band of semiconductors alloys were performed employing density functional theory (DFT) with local density approximation (LDA) [8] for exchange and correlation with parameterization of Perdew and Zunger [9]. The electron-ion interaction has been described employing relativistic separable Kleinman-Bylander pseudopotentials [10, 11]. This computational scheme provides a very accurate description of the spin splitting in various semiconductors $[11,12]$.

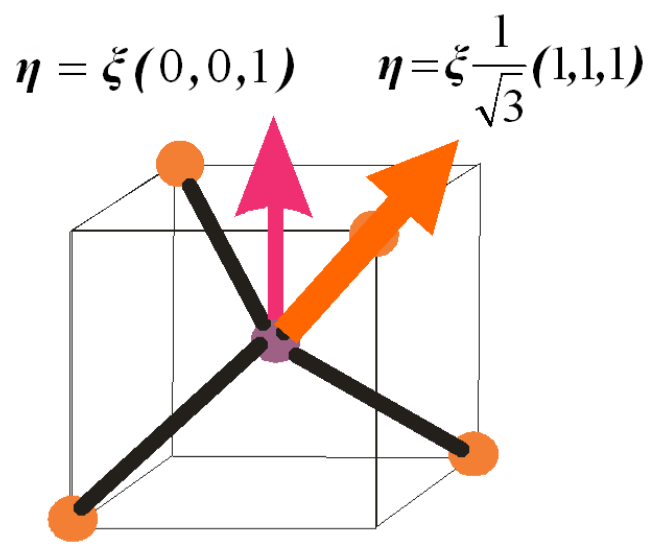

Fig. 1. The nearest surrounding of an atom in the strain deformed unit cell. One atom is placed at the origin. The second atom can move along $\boldsymbol{\eta}$ direction without changing the crystal symmetry. The invariant directions, for $C_{2 v}$ symmetry $\boldsymbol{\eta}=\xi(0,0,1)$ and for $C_{3 v}$ symmetry $\boldsymbol{\eta}=\xi \frac{1}{\sqrt{3}}(1,1,1)$, are indicated in the figure. The position of the second atom in the unit cell is determined by the minimization of the total energy. 
The deformation of the cubic lattice is described by deformation (strain) tensor

$$
\hat{\varepsilon}=\left(\begin{array}{lll}
\varepsilon_{x x} & \varepsilon_{x y} & \varepsilon_{x x} \\
\varepsilon_{y x} & \varepsilon_{y y} & \varepsilon_{y z} \\
\varepsilon_{z x} & \varepsilon_{z y} & \varepsilon_{z z}
\end{array}\right) .
$$

The deformation tensor can be in general non-symmetric. The vectors of primitive translations in the strained crystal are modified by the lattice deformation $\boldsymbol{a}_{i}^{\prime}=$ $(1+\hat{\varepsilon}) \boldsymbol{a}_{i}$, where $\left\{\boldsymbol{a}_{i}\right\}$ are fcc lattice vectors, whereas the basis vectors of the strained crystal take the following shape $\boldsymbol{\tau}_{\alpha}^{\prime}=(1+\hat{\varepsilon}) \boldsymbol{\tau}_{\alpha}+\boldsymbol{\eta}$, where $\left\{\boldsymbol{\tau}_{\alpha}\right\}$ are the basis vectors of the unstrained crystal, and $\boldsymbol{\eta}$ is the shift of the atoms along the line that remains invariant under symmetry operations of the point group of the strained crystal (see Fig. 1). This $\boldsymbol{\eta}$ is usually called the internal strain parameter and can be only determined by the minimization of the total energy of the strained crystal. The form of the deformation tensor, determines the reduced symmetry (from $T_{d}$ ) of the strained lattice, for example

$$
\begin{aligned}
& \left(\begin{array}{ccc}
0 & \varepsilon & 0 \\
\varepsilon & 0 & 0 \\
0 & 0 & 0
\end{array}\right) \rightarrow C_{2 v}\left(\begin{array}{ccc}
0 & \varepsilon & \varepsilon \\
\varepsilon & 0 & \varepsilon \\
\varepsilon & \varepsilon & 0
\end{array}\right) \rightarrow C_{3 v}\left(\begin{array}{ccc}
0 & \varepsilon & 0 \\
0 & 0 & 0 \\
0 & 0 & 0
\end{array}\right) \\
& \quad \rightarrow C_{2}\left(\begin{array}{ccc}
0 & \varepsilon & 0 \\
-\varepsilon & 0 & 0 \\
0 & 0 & 0
\end{array}\right) \rightarrow S_{4} .
\end{aligned}
$$

We have considered various lattice deformations (also described by non-symmetric tensors), and for each of them we have calculated the total energies, the optimized geometry of the unit cell (just determining the internal strain parameter $\boldsymbol{\eta}$ ) and further calculated the relativistic band structure of the strained semiconductor. This procedure allows us to calculate conduction band spin splitting that will be analyzed in the next section within the effective Hamiltonian.

\section{Results - linear- $k$ spin splitting in conduction band}

Traditionally the strain induced spin splitting of the conduction band is described by the Pikus and Titkov Hamiltonian [7]. Since the strain reduces the crystal symmetry, the linear- $k$ terms in the Hamiltonian become allowed

$$
\hat{H}_{\mathrm{SO}}^{(\mathrm{PT})}(\hat{\varepsilon}, \boldsymbol{k})=\boldsymbol{\sigma} \cdot\left[\boldsymbol{\Omega}_{\mathrm{R}}^{(\mathrm{PT})}(\hat{\varepsilon}, \boldsymbol{k})+\boldsymbol{\Omega}_{\mathrm{D}}^{(\mathrm{PT})}(\hat{\varepsilon}, \boldsymbol{k})\right],
$$

where $\boldsymbol{\sigma}$ is the vector of the Pauli matrices and effective magnetic fields $\boldsymbol{\Omega}_{\mathrm{R}}^{(\mathrm{PT})}$ and $\boldsymbol{\Omega}_{\mathrm{D}}^{(\mathrm{PT})}$ read

$$
\begin{aligned}
& \Omega_{\mathrm{R} x}^{(\mathrm{PT})}=C_{3}\left(\varepsilon_{z x} k_{z}-\varepsilon_{x y} k_{y}\right), \quad \Omega_{\mathrm{R} y}^{(\mathrm{PT})}=C_{3}\left(\varepsilon_{x y} k_{x}-\varepsilon_{y z} k_{z}\right), \\
& \Omega_{\mathrm{R} z}^{(\mathrm{PT})}=C_{3}\left(\varepsilon_{y z} k_{y}-\varepsilon_{z x} k_{x}\right), \quad \Omega_{\mathrm{D} x}^{(\mathrm{PT})}=D\left(\varepsilon_{y y}-\varepsilon_{z z}\right) k_{x}, \\
& \Omega_{\mathrm{D} y}^{(\mathrm{PT})}=D\left(\varepsilon_{z z}-\varepsilon_{x x}\right) k_{y}, \quad \Omega_{\mathrm{D} z}^{(\mathrm{PT})}=C_{3}\left(\varepsilon_{x x}-\varepsilon_{y y}\right) k_{z} .
\end{aligned}
$$


The constants $C_{3}$ and $D$ may be calculated using perturbation theory or fitted, at least in principle, to the values of spin splitting obtained in the ab initio calculations. In this section, we concentrate on deformations characterized by tensors with zero diagonal elements as exemplified in Eq. (2). We compare the conduction band spin splitting obtained from the ab initio calculations with the spin splitting calculated from the diagonalization of the effective Pikus-Titkov Hamiltonian (Eq. (3)). This comparison leads to the conclusion that the Pikus-Titkov Hamiltonian generally does not describe spin splitting obtained from the first principles.

The first-principle calculations clearly show that there is no linear- $k$ spin splitting for the deformation reducing $T_{d}$ symmetry to $S_{4}$ (tensor No. 4 in Eq. (2)), whereas Hamiltonian of Pikus and Titkov leads to such splitting. Further, the spin splitting for the symmetry $C_{2}$ as calculated from the ab initio is only half as large as obtained from the Pikus-Titkov Hamiltonian. It is very well understood, if one writes the deformation tensor leading to the $C_{2}$ symmetry as

$$
\left(\begin{array}{lll}
0 & \varepsilon & 0 \\
0 & 0 & 0 \\
0 & 0 & 0
\end{array}\right)=\left(\begin{array}{ccc}
0 & \varepsilon / 2 & 0 \\
\varepsilon / 2 & 0 & 0 \\
0 & 0 & 0
\end{array}\right)+\left(\begin{array}{ccc}
0 & \varepsilon / 2 & 0 \\
-\varepsilon / 2 & 0 & 0 \\
0 & 0 & 0
\end{array}\right) .
$$

Since the second part of the deformation tensor does not induce the linear- $k$ spin splitting, the spin splitting is determined solely by the first part of the deformation tensor. In general, the Pikus-Titkov Hamiltonian describes correctly the conduction band spin splitting for symmetric deformation tensors only, $\hat{\varepsilon}_{\alpha \beta}=\hat{\varepsilon}_{\beta \alpha}$. Then, the Pikus-Titkov Hamiltonian is equivalent to the Rashba Hamiltonian

$$
\hat{H}_{\mathrm{SO}}^{(\mathrm{PT})}(\boldsymbol{k})=\hat{H}_{\mathrm{eff}}^{\mathrm{Rashba}}(\boldsymbol{k})=\boldsymbol{\sigma} \cdot(\boldsymbol{k} \times \boldsymbol{n}),
$$

where the vector $\boldsymbol{n}=\left(\varepsilon_{y z}, \varepsilon_{x z}, \varepsilon_{x y}\right)$ determines the axis of uniaxial symmetry.

In the case of deformations leading to symmetry $C_{2 v}$ and $C_{3 v}$, the corresponding expressions for the spin splitting obtained from the Pikus-Titkov Hamiltonian read respectively,

$$
\Delta E_{\uparrow \downarrow}^{(\mathrm{PT})}(\boldsymbol{k})=2 C_{3}|\varepsilon| \sqrt{k_{x}^{2}+k_{y}^{2}}
$$

and

$$
\Delta E_{\uparrow \downarrow}^{(\mathrm{PT})}(\boldsymbol{k})=2 C_{3}|\varepsilon| \sqrt{\left(k_{y}-k_{z}\right)^{2}+\left(k_{z}-k_{x}\right)^{2}+\left(k_{x}-k_{y}\right)^{2}} .
$$

In the case of $C_{2 v}$ symmetry, the in-plane spin splitting (i.e., for wave vectors lying in the $x-y$ plane) obtained from the Pikus-Titkov Hamiltonian is isotropic. It is not the case of spin splitting obtained from the first-principles calculation, where for this symmetry the in-plane spin splitting shows a clear anisotropy. Additionally, as can be seen from Eq. (8), the spin splitting obtained from the Pikus-Titkov Hamiltonian for crystal structure of $C_{3 v}$ does not depend on the sign of the deformation. This contradicts the $a b$ initio calculations, which lead to the spin splitting such that $\Delta E_{\uparrow \downarrow}^{(\text {ab-initio })}(-\varepsilon, \boldsymbol{k}) \neq \Delta E_{\uparrow \downarrow}^{(\text {ab-initio })}(\varepsilon, \boldsymbol{k})$. All this clearly demonstrates that another effective Hamiltonian is needed to describe the linear- $k$ conduction band spin splitting from the first-principles calculations. 
Here, we construct such an effective Hamiltonian and describe microscopic nature of the parameters entering the Hamiltonian. The effective Hamiltonian has the form

$$
\hat{H}_{\mathrm{eff}}(\boldsymbol{k})=\boldsymbol{\sigma} \cdot\left[\boldsymbol{\Omega}_{\mathrm{R}}(\boldsymbol{k})+\boldsymbol{\Omega}_{\mathrm{D}}(\boldsymbol{k})\right],
$$

where $\boldsymbol{\Omega}_{\mathrm{R}}(\boldsymbol{k})$ and $\boldsymbol{\Omega}_{\mathrm{D}}(\boldsymbol{k})$ are the effective magnetic fields. For $C_{2 v}$ symmetry,

$$
\boldsymbol{\Omega}_{\mathrm{R}}(\boldsymbol{k})=\alpha_{\mathrm{R}}\left(k_{y},-k_{x}, 0\right) \text { and } \boldsymbol{\Omega}_{\mathrm{D}}(\boldsymbol{k})=\alpha_{\mathrm{D}}\left(-k_{x}, k_{y}, 0\right),
$$

whereas for $C_{3 v}$ symmetry

$$
\boldsymbol{\Omega}_{\mathrm{R}}(\boldsymbol{k})=\alpha_{\mathrm{R}} \frac{1}{\sqrt{3}}\left(k_{y}-k_{z}, k_{z}-k_{x}, k_{x}-k_{y}\right) \quad \text { and } \quad \boldsymbol{\Omega}_{\mathrm{D}}(\boldsymbol{k})=\frac{\alpha_{\mathrm{D}}}{\alpha_{\mathrm{R}}} \boldsymbol{\Omega}_{\mathrm{R}}(\boldsymbol{k}),
$$

where $\alpha_{\mathrm{R}}$ and $\alpha_{\mathrm{D}}$ are materials constant. The spin splitting for $C_{2 v}$ and $C_{3 v}$ symmetry is equal to

$$
\begin{aligned}
& \Delta E_{\uparrow \downarrow}^{\left(C_{2 v}\right)}=2 \boldsymbol{k}_{\|} \sqrt{\alpha_{\mathrm{R}}^{2}+\alpha_{\mathrm{D}}^{2}-2 \alpha_{\mathrm{D}} \alpha_{\mathrm{R}} \sin 2 \varphi}, \\
& \Delta E_{\uparrow \downarrow}^{\left(C_{3 v}\right)}=2 \boldsymbol{k}\left|\alpha_{\mathrm{R}}+\alpha_{\mathrm{D}}\right| \sqrt{\left(k_{y}-k_{z}\right)^{2}+\left(k_{z}-k_{x}\right)^{2}+\left(k_{x}-k_{y}\right)^{2}},
\end{aligned}
$$

where $\varphi$ is the angle between in-plane wave vector $\boldsymbol{k}_{\|}$(i.e., lying in the $(x, y)$ plane) and the [100] cubic direction.

The $a b$ initio studies allow for relating the constants $\alpha_{\mathrm{R}}$ and $\alpha_{\mathrm{D}}$ in the effective Hamiltonian to the strength of the crystal deformation $\varepsilon$ and additionally to internal strain parameter (i.e., cell geometry). The analysis of spin splitting obtained from the first principles gives us the proper form of the constants $\alpha_{\mathrm{R}}$ and $\alpha_{\mathrm{D}}$

$$
\alpha_{\mathrm{R}}=\alpha(\xi) \varepsilon, \quad \alpha_{\mathrm{D}}=\beta(\xi) \varepsilon^{2},
$$

where $\xi$ determines the position of the second atom in the deformed unit cell along the invariant direction $\boldsymbol{\eta}$ and takes a special value $\xi_{0}$ for the optimized unit cell geometry. Let us note that the internal strain parameter $\xi_{0}$ depends itself on the external strain $\varepsilon$. It is now completely clear (see Eqs. (10)), why the spin splitting is not isotropic for the in-plane wave vectors in the case of the $C_{2 v}$ symmetry (simply $\alpha_{\mathrm{D}}$ is not zero) and a change of the sign in the deformation tensor $(\varepsilon \rightarrow-\varepsilon)$ leads to a different magnitude of the spin splitting in the case of $C_{3 v}$ symmetry. In this manner, we have constructed the effective Hamiltonian that describes the linear- $k$ conduction band spin splitting and reproduces the linear- $k$ spin splitting obtained from the first principles. This effective Hamiltonian involves terms that are linear in the wave vector $\boldsymbol{k}$ and quadratic in the deformation strength $\varepsilon$. Since the latter are lacking in the Pikus-Titkov Hamiltonian, the richer structure of the linear- $k$ spin splitting cannot be reproduced with it. One can say that the PikusTitkov Hamiltonian approximates the linear- $k$ spin splitting up to terms linear in the strain. 


\section{Conclusions}

The first-principles calculations reveal the physical mechanisms that determine the strain induced linear- $k$ spin splitting. They show that the terms quadratic in strain play an important role and neglecting them leads to not correct structure of spin splitting. It turns out that the strain induced linear- $k$ spin splitting in III-V semiconductors is dependent on the geometry of the deformed unit cell. Therefore, the strain induced linear- $k$ spin splitting can be determined accurately only by means of the first-principles calculations.

\section{References}

[1] Semiconductor Spintronics and Quantum Computation, Eds. D.D. Awschalom, N. Samarth, D. Loss, Springer-Verlag, Berlin 2002.

[2] I. Žutić, J. Fabian, S. Das Sarma, Rev. Mod. Phys. 76, 323 (2004).

[3] S. Murakami, N. Nagaosa, S. Zhang, Science 301, 1348 (2003); L.S. Levitov, E. Rashba, Phys. Rev. B 67, 115324 (2003); C.S. Tang, A.G. Mal'shukov, K.A. Chao, Phys. Rev. B 71, 195314 (2005).

[4] J. Wunderlich, B. Käestner, J. Sinova, T. Jungwirth, Phys. Rev. Lett. 94, 047204 (2005); Y.K. Kato, R.C. Myers, A.C. Gossard, D.D. Awschalom, Appl. Phys. Lett. 87, 022503 (2005).

[5] Y.K. Kato, R.C. Myers, A.C. Gossard, D.D. Awschalom, Phys. Rev. Lett. 93, 176601 (2004).

[6] A.G. Mal'shukov, C.S. Tang, C.S. Chu, K.A. Chao, Phys. Rev. Lett. 95, 107203 (2005).

[7] G.E. Pikus, A.N. Titkov, in: Optical Orientation, Eds. F. Meier, B.P. Zakharchenya, Vol. 8, North-Holland, Amsterdam 1984, p. 73.

[8] W. Kohn, L.J. Sham, Phys. Rev. A 140, 1133 (1965).

[9] J.P. Perdew, A. Zunger, Phys. Rev. B 23, 5048 (1981).

[10] B.M. Bylander, L. Kleinman, Phys. Rev. B 41, 7868 (1990).

[11] J.A. Majewski, S. Birner, A. Trellakis, M. Sabathil, P. Vogl, Phys. Status Solidi C 1, 2003 (2004).

[12] J.A. Majewski, M. Städele, P. Vogl, Mater. Res. Soc. Symp. Proc. 449, 887 (1997); J.A. Majewski, P. Vogl, in: Physics of Semiconductors 2002, Proc. 26th Int. Conf. Phys. Semicond., Edinburgh 2002, Eds. A.R. Long, J.H. Davies, Institute of Physics Publishing, Bristol 2003, p. 305. 